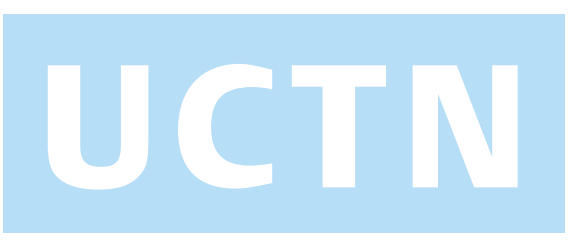

\title{
Use of a Duodenoscope in the Management of a Ureteral Calculus in a Patient with Ureterosigmoid- ostomy (Mainz Pouch Il; Rectosigmoid Pouch)
}

Mainz pouch II (modified ureterosigmoidostomy) is an established technique for permanent urinary diversion in patients with congenital malformations or acquired diseases of the bladder [1]. If urolithiasis occurs, an effective and minimally invasive therapy is mandatory. Extracorporeal shock-wave lithotripsy and surgical procedures are frequently used treatment strategies [2]. Retrograde endoscopic management is an alternative option, which has been reported rarely.

We report here the case of a 24-year-old man, who had undergone ureterosigmoidostomy at the age of 12 months due to bladder exstrophy. The follow-up was unremarkable until the patient's current presentation with acute right flank pain. The ultrasound examination revealed right-sided hydronephrosis, and computed tomography identified a 6-mm ureteral calculus. During sigmoidoscopy, both ureteral ostia were located at a distance of $15 \mathrm{~cm}$ from the anal verge, with a normal macroscopic appearance. Urine drainage was identified only at the proximal ostium (Figure 1). The "dry" distal ostium was therefore intubated using a duodenoscope with a conventional endoscopic retrograde cholangiopancreatography (ERCP) catheter. A retrograde ureterogram was obtained by injecting contrast medium, revealing the $6-\mathrm{mm}$ concrement in the distal ureter (Figure 2). A Dormia basket was inserted and the calculus extracted (Figure 3 ).

To our knowledge, this is the first reported case of successful extraction of a ureteral calculus using a duodenoscope in a patient with Mainz pouch II. Fitzgerald et al. report successful management of a ureteral calculus in a patient with ureterosigmoidostomy by retrograde sigmoid endoscopy with a 21-Fr cystoscope, in combination with intracorporeal lithotripsy [3]. Costamagna et al. successfully managed 19 of 24 ureteroileal complications in patients with ileal pouches using a duodenoscope. A ureteral calculus was removed in one patient [4].

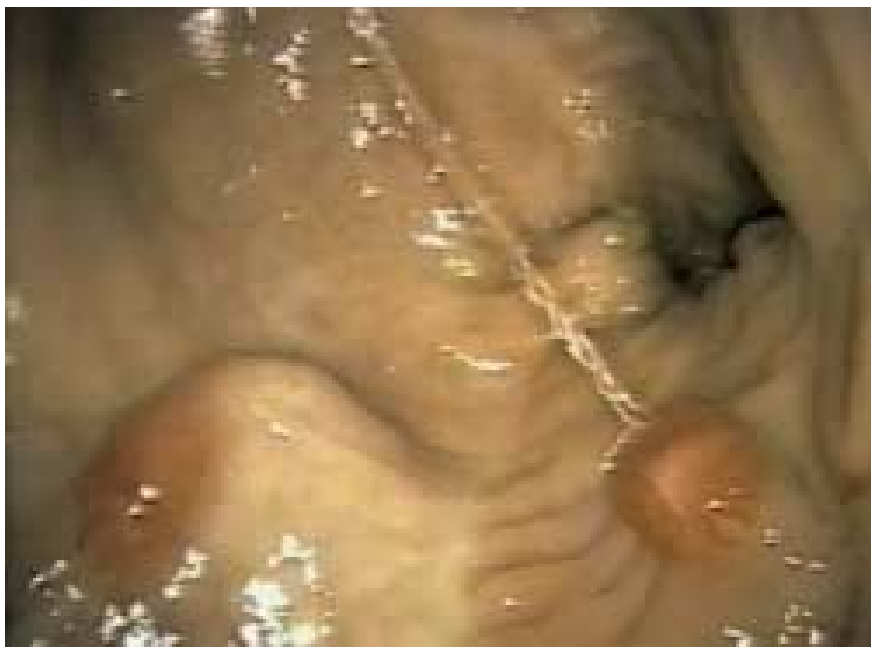

Figure 1 Endoscopic image of the proximal ureteral ostium, with urine draining into the Mainz pouch II.

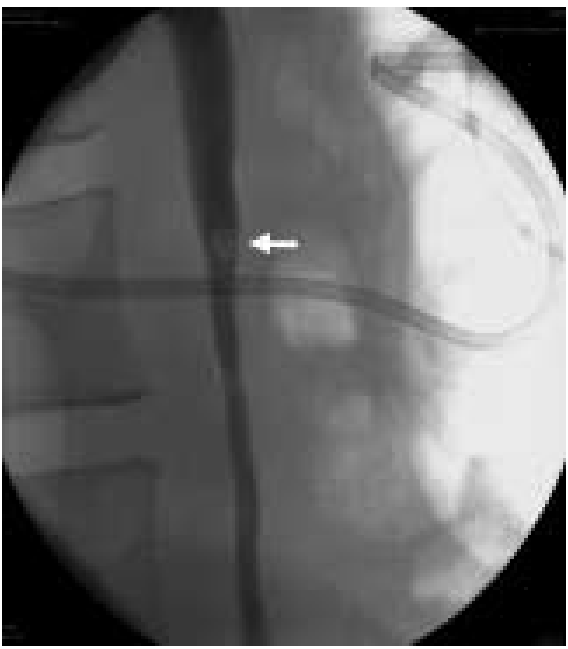

Figure 2 The retrograde ureterogram, obtained by injecting contrast medium after intubating the ostium with an endoscopic retrograde cholangiopancreatography catheter, shows the ureteral calculus.

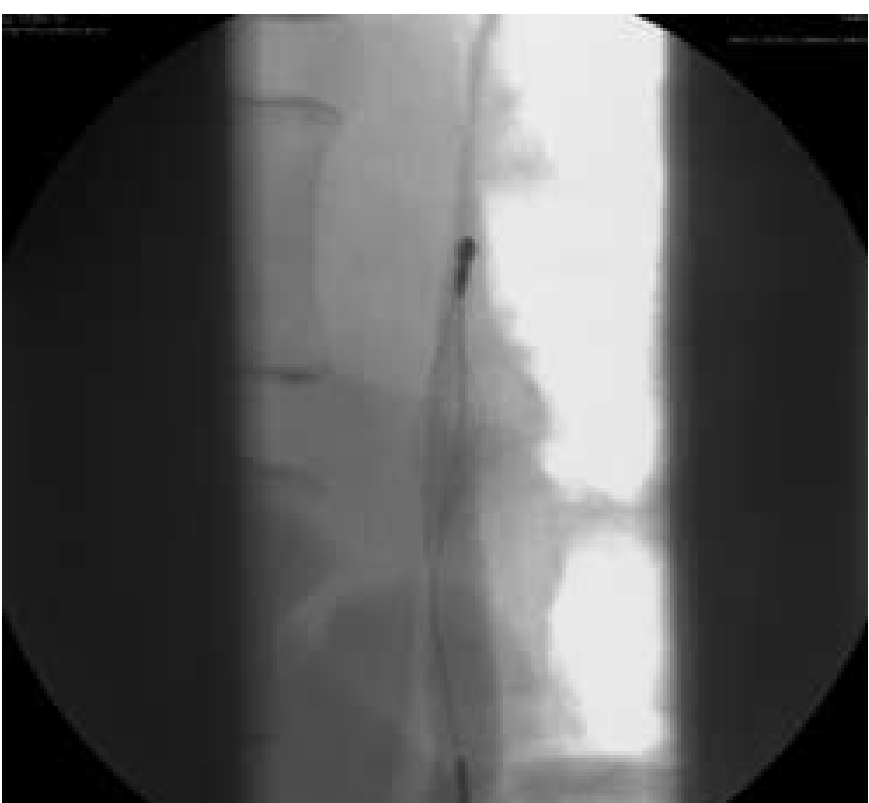

Figure 3 Retrograde ureterogram, showing the Dormia basket in the ureter. 
Retrograde endoscopic management of ureteral calculi is thus a simple technique, which is minimally invasive and provides good access to the distal ureter in patients with ureterosigmoidostomy.

\section{P. Mosler ${ }^{1}$ R. Kiesslich ${ }^{1}$, R. Stein ${ }^{2}$,} P. R. Galle ${ }^{1}$, J. W. Thüroff ${ }^{2}$, S. Kanzler ${ }^{1}$

${ }^{1}$ Dept. of Medicine I, Johannes Gutenberg University, Mainz, Germany

2 Dept. of Urology, Johannes Gutenberg University, Mainz, Germany.

\section{References}

${ }^{1}$ Fisch M, Wammack R, Müller S, Hohenfellner R. The Mainz pouch II (sigma rectum pouch). J Urol 1993; 149: 258 263

${ }^{2}$ Cohen T, Streem SB, Lammert GK. Selective minimally invasive management of calculi in patients with urinary diversions. J Urol 1994; 152: 1091 - 1094

${ }^{3}$ Fitzgerald KB, Aslan P, Preminger GM. Endourological management of a large distal ureteral calculus in a patient with ureterosigmoidostomy diversion. J Urol 1998; 159: 2081 - 2082

${ }^{4}$ Costamagna G, Shah SK, Mutignani M et al. Use of a duodenoscope to manage complications at the ureteroileal anastomotic site after total urinary bladder resection and the Bricker procedure. Gastrointest Endosc 2002; 55: $242-$ 248
Corresponding Author

\section{P. Mosler, M.D.}

I. Medizinische Klinik und Poliklinik Johannes-Gutenberg-Universität Langenbeckstrasse 1 55101 Mainz - Germany Fax: $\quad+49-6131-175552$ E-mail: patrickmosler@web.de 